\title{
Avaliação do grau de funcionalidade de pacientes que sofreram acidente vascular encefálico antes e após intervenção fisioterapêutica no solo e na hidroterapia Functional assessment of neurological patients with stroke, before and after physical therapy and hidrotherapy
}

\author{
Carina Tárzia K akihara*, Cristiane G aspar N eves** \\ * Supervisora do Estágio de F isi oterapia N eurológica (Solo) da U niversidadePaulista, Campus A nchieta, São Paulo, \\ **Supervisora do Estágio de F isioterapia N eurológica (H idroterapia) da U niversidadePaulista, Campus A nchieta, São Paulo
}

\section{Resumo}

O acidente vascular encefálico (AVE) é caracterizado pela instalação de um déficit neurológico focal, repentino, não convulsivo, determinado por uma lesão cerebral secundária a um mecanismo vascular. O paciente que sofre um AVE pode apresentar postura e movimentos anormais e, com isso, há um grande risco de desenvolver deformidade estrutural e limitação nas atividades de vida diária (AVD). Este trabalho propõe-se a comparar o grau de funcionalidade de pacientes após AVE, antes e após a intervenção fisioterapêutica no solo, com ou sem a hidroterapia. Como resultado, foi identificada melhora dos pacientes que fizeram hidroterapia nas seguintes variáveis: asseio,micção, vestir, mobilidade e escadas. $\mathrm{N}$ os pacientes que não fizeram hidroterapia, houve melhora nas seguintes variáveis: evacuação, micção, cadeira/ cama, escadas e banho.

Conclui-se que mesmo em um curto período de tempo, a fisioterapia no solo e a hidroterapia auxiliam na melhora da realização das AVD s nos pacientes com AVE e que, sempre que possível, a hidroterapia deve ser sugerida como terapia complementar.

Palavras-chave: fisioterapia no solo, hidroterapia, acidente vascular encefálico, atividade de vida diária, escala de Barthel

\begin{abstract}
The stroke is characterized by installation of a sudden, non convulsive and focal neurological deficiency, determined by a secondary vascular brain damage mechanism. The patient with stroke may show abnormal posture and movement, and therefore an important risk of structural deformities development and limitation on daily activities. The aim of this study was to compare the stroke patients functionality degree, before and after physical therapy on ground, with or without hidrotherapy. As a result, it was identified improvement of patients with hidrotherapy in the following variables: cleaness, urination, dress, mobility and stairs. A bout the patients without hidrotherapy, it was identified improvement about following variables: evacuation, urination, chair/ bed, stairs and bath. It can be concluded that even in brief time, physical therapy on ground and hidrotherapy help in improvement of realization of daily activities for patients with stroke, and that, always when possible, hidrotherapy must be suggested as a complementary therapy.
\end{abstract}

Key-words: physical therapy on ground, hidrotherapy, stroke, daily activities, Barthel index.

\section{Introdução}

0 acidente vascular encefálico (AVE) é definido como início abrupto, ou em forma de crise, de sintomas neurológicos focais ou globais causados por isquemia ou hemor- ragia no cérebro ou a sua volta, em conseqüência de doenças de vasos sangüíneos cerebrais. Pelas definições clínicas convencionais, diagnostica-se acidente vascular encefálico em uma pessoa se os sintomas continuam por mais de 24 horas [1].

N os pacientes sobreviventes ao primeiro mês, cerca de $10 \%$ têm cura espontânea, 10\% ficam severamente incapacitados, 
com alteração persistente do estado de consciência e os $80 \%$ restantes ficam com disfunção neurológica, maior ou menor, necessitando de reabilitação para diminuir seu estado de dependência e prevenir complicações [2].

$\mathrm{U} \mathrm{m}$ dos comprometimentos motores evidentes na hemiplegia é a tendência em manter-se em uma posição de assimetria postural, com distribuição de peso menor sobre o lado afetado e conseqüente transferência do peso corporal para o lado oposto. Essa assimetria e dificuldade em suportar o peso no lado afetado interferem na capacidade de manter 0 controle postural, impedindo a orientação e estabilidade para realizar movimentos com o tronco e membros. Cabe ressaltar que a execução das atividades de vida diária, tais como: vestir-se, alimentar-se, mudar de posição, andar, alcançar objetos dependem e envolvem esse controle postural. No caso do hemiplégico manter uma atitude postural que desloca o plano de distribuição do centro de gravidade e altera a superfície de suporte, estabelece-se uma condição de instabilidade e de desequilíbrio que interfere no desempenho das atividades funcionais e prejudica a execução das tarefas da vida diária [3].

Tarefas de autocuidado são atividades da vida diária e de rotina, necessárias para sobreviver. Essas tarefas dependem da idade, gênero e meio ambiente de cada pessoa. Exemplos dessas atividades são: vestir, comer, tomar banho, arrumarse, toalete e mobilidade dentro de casa. Se a performance dessas atividades é dificultada ou não é possível, está presente uma incapacidade. Se a falta de habilidade para realizar essas atividades resulta em desvantagem social, a condição é vista como uma deficiência física [4].

$D$ ificuldades na realização das $A V D_{s}$ e a dependência de outros para completar essas tarefas podem provocar um efeito devastador nos aspectos psicológico, social e financeiro dos pacientes. D epressão, diminuição da auto-estima e perda da motivação podem resultar no fracasso da reabilitação desses pacientes, enquanto que a melhora na realização dessas $A V D_{s}$ pode conduzir a um aumento da auto-estima [5-7].

Bartels [8] sugere que quando uma pessoa é independente nas $A V D_{s}$ e quando ela se mostra incapaz de realizar estas $A V D_{s}$ devido a uma patologia, a situação muda completamente. Independência nas AVD ajuda a rebater a idéia de que a pessoa com uma incapacidade pode ser um fardo financeiro ou social para a sociedade. Bartels nota que uma incapacidade somente se torna um fardo para a realização de alguma atividade, quando assim é considerado pelo paciente perante outras pessoas ou quando o meio ambiente faz isso também.

O Índice de Barthel foi desenvolvido para monitorar independência funcional em pacientes que necessitam de reabilitação a longo prazo e é utilizado extensivamente em pesquisas como uma medida das $A V D_{s^{\prime}}$ Ele é simples de administrar, e a credibilidade e validade dos dados obtidos com esse índice é provado em vários estudos com uma variedade de populações de pacientes [9-13].
E m 1965, M ahoney e Barthel publicaram uma escala importante para mensurar $A V D_{s}$ básicas com pacientes com incapacidades crônicas. D escrito como "um simples índice de independência para pontuar a habilidade do próprio paciente com uma desordem neuromuscular ou músculoesquelética", o Índice de Barthel inclui 10 itens, como alimentação, transferência, arrumar-se e higiene, banho, toalete, andar, transpor degraus, e controle do intestino e bexiga. I tens são pontuados diferentemente, de acordo com um sistema de pontuação que marca pontos baseados na realização independente ou com auxílio. U m paciente com um escore máximo de 100 pontos é definido como continente, capaz de se alimentar e vestir-se de forma independente, de andar pelo menos um quarteirão, e subir e descer degraus. Os autores foram cuidadosos para notar que um escore máximo não significa necessariamente independência, mesmo porque AVD ${ }_{5}$ como cozinhar, limpar casa e socialização não são avaliadas [4].

Considerando as alterações nas AVD s após AVE e a necessidade de uma abordagem multidisciplinar para uma recuperação funcional de pacientes que foram acometidos por AVE, julgou-se relevante um estudo cujo objetivo é comparar o grau de funcionalidade de pacientes após AVE, antes e após a intervenção fisioterapêutica em 2 grupos de pacientes: um grupo de pacientes que só realizou fisioterapia no solo e outro onde os pacientes fizeram fisioterapia no solo mais a hidroterapia, em um período de 2 meses de tratamento.

\section{Material e métodos}

\section{Sujeitos}

0 presente estudo foi realizado com 11 pacientes com diagnóstico de AVE isquêmico ou hemorrágico, de ambos os sexos, com idade entre 53 e 81 anos, e encaminhados para a Clínica de Fisioterapia da U niversidade Paulista, no Campus A nchieta, no período de outubro a dezembro de 2003. D esse total de pacientes, todos realizaram a fisioterapia no solo, sendo que apenas 5 realizaram hidroterapia em associação à terapia do solo.

Cada paciente realizou a fisioterapia no solo, 2 a 3 vezes semanalmente e na água, 1 vez por semana. A sessão no solo teve duração de 50 minutos e cada terapia na água durava 40 minutos.

Os pacientes ou responsáveis foram informados que eles poderiam ser selecionados para participar desse presente estudo. A pós os esclarecimentos, os pacientes ou responsáveis assinaram termo de consentimento para autorizar a participação do paciente nesse trabalho.

\section{Procedimentos}

Inicialmente, realizou-se uma triagem de cada paciente, constituída por uma anamnese. Então, o paciente era 
encaminhado para realizar a fisioterapia no solo e a hidroterapia, se houvesse indicação específica para esta última abordagem terapêutica, disponibilidade de horário, liberação dermatológica e se o paciente tivesse controle do esfíncter vesical e anal. A pós a avaliação completa de cada paciente, a supervisora responsável completava a ficha do Índice de Barthel, onde o paciente, ou o responsável, respondia às perguntas referentes à esse Índice (Tabela I).

Tabela I - Índicede Barthel.

\begin{tabular}{|c|c|c|}
\hline Atividade & ação opcional & Critérios \\
\hline V1-Alimentação & 1050 & $\begin{array}{l}\text { Independente } \\
\text { N ecessita ajuda } \\
\text { Dependente }\end{array}$ \\
\hline V2-Asseio & 50 & $\begin{array}{l}\text { Independente } \\
\text { Dependente }\end{array}$ \\
\hline V3-Evacuação & 1050 & $\begin{array}{l}\text { Continente } \\
\text { Acidente ocasional } \\
\text { Incontinente }\end{array}$ \\
\hline V4-Micção & 1050 & $\begin{array}{l}\text { Continente } \\
\text { Acidente ocasional } \\
\text { Incontinente }\end{array}$ \\
\hline V5-Vestir & 1050 & $\begin{array}{l}\text { Independente } \\
\text { Necessita ajuda } \\
\text { Dependente }\end{array}$ \\
\hline V6-C adeira/Cama & 151050 & $\begin{array}{l}\text { Independente } \\
\text { Ajuda mínima } \\
\text { Capaz de sentar } \\
\text { Dependente }\end{array}$ \\
\hline V7-Toalete & 1050 & $\begin{array}{l}\text { Independente } \\
\text { N ecessita ajuda } \\
\text { Dependente }\end{array}$ \\
\hline V8-Mobilidade & 151050 & $\begin{array}{l}\text { Independente } \\
\text { Ajuda mínima } \\
\text { Independente em cadeira } \\
\text { de rodaslmóvel }\end{array}$ \\
\hline V9-Escadas & 1050 & $\begin{array}{l}\text { Independente } \\
\text { N ecessita ajuda } \\
\text { Incapaz }\end{array}$ \\
\hline V10-Banho & 50 & $\begin{array}{l}\text { Independente } \\
\text { Dependente }\end{array}$ \\
\hline
\end{tabular}

V11- Total (máximo 100 pontos)-

Pontuação Total Final:

Fonte: Mahoney e Barthel (1965).

U ma vez avaliados, os pacientes foram submetidos a tratamento fisioterapêutico, seguindo os métodos de facilitação neuromuscular proprioceptiva (FNP) e conceitos do método Bobath. A pós 2 meses de tratamento, os pacientes responderam novamente o Índice de Barthel para a mesma supervisora responsável.

A proposta de tratamento fisioterapêutico no solo e na água visou: amenizar ou normalizar as seqüelas da lesão cerebral, como por exemplo: hipertonia, fraqueza muscular e amplitude de movimento, além de fornecer um ambiente estável para a participação ativa do paciente na melhora da habilidade funcional.
Para isso, no solo utilizou-se a abordagem baseada nos conceitos do $\mathrm{K}$ abat e do Bobath. $\mathrm{Na}$ água, a proposta de tratamento foi baseada na utilização dos efeitos físicos, fisiológicos e cinesiológicos provenientes da imersão do corpo ou parte dele, além de técnicas específicas de reabilitação aquática, como Watsu, Halliwick e Bad Ragaz.

A partir dos resultados do Índice de Barthel, antes e após a fisioterapia no solo e hidroterapia, foi feita uma análise comparativa e descritiva dos mesmos.

\section{M étodo estatístico}

Com a finalidade de avaliarmos possíveis diferenças entre os escores avaliados no mês de dezembro em relação ao mês de outubro, para as variáveis previamente definidas, tanto no grupo sem terapia na água como no grupo com terapia na água, usamos o teste não paramétrico para 2 amostras não independentes de W ilcoxon [14].

Para avaliarmos possíveis diferenças entre os grupos para cada variável no final da observação, isto é, em dezembro, preferimos definir a variável delta \%:

$$
\begin{gathered}
\Delta \%=\text { Escore dez - escore out } \times 100 \\
\text { Escore out }
\end{gathered}
$$

A razão desta variável é que, avaliando o valor absoluto dos escores em dezembro, podemos ter valores iguais, que em outubro eram diferentes entre si e, portanto, um mesmo

valor em dezembro não tem o mesmo significado, pois ele é uma função do valor que havia em outubro.

0 nível de rejeição para a hipótese de nulidade foi fixado sempre em um valor igual ou menor do que 0,05 (5\%).

As médias foram calculadas e apresentadas a título de informação.

$\mathrm{N}$ ão foram calculados desvios-padrão pois, usando testes não paramétricos, estamos pressupondo que as variáveis em causa não se comportam como curva de Gauss e, portanto, não há sentido o seu cálculo.

\section{Resultados}

A partir dos escores obtidos com o Índice de Barthel, no mês de outubro e dezembro, dos pacientes que não realizaram hidroterapia $(\mathrm{N}=0)$, temos os seguintes valores das variáveis:

Tabela II - R esultados do Í ndicedeBarthel para cada variável em outubro e dezembro dos pacientes que não fizeram hidroterapia.

\begin{tabular}{lcccccc}
\hline $\begin{array}{l}\text { Pacientes } \\
\text { Variá veis }\end{array}$ & $\mathbf{P 1}$ & $\mathbf{P 2}$ & $\mathbf{P 6}$ & $\mathbf{P 8}$ & $\mathbf{P ~ 9}$ & $\mathbf{P 1 0}$ \\
\hline O u1 & 5 & 5 & 10 & 5 & 5 & 5 \\
D v1 & 5 & 5 & 10 & 5 & 5 & 5 \\
O v2 & 5 & 0 & 5 & 0 & 0 & 5 \\
D V2 & 5 & 0 & 5 & 0 & 0 & 5 \\
O v3 & 10 & 5 & 10 & 0 & 10 & 5 \\
D v3 & 10 & 10 & 10 & 0 & 10 & 5 \\
O v4 & 0 & 0 & 10 & 0 & 5 & 5
\end{tabular}




\begin{tabular}{lcccccc}
\hline $\begin{array}{l}\text { Pacientes } \\
\text { Variá veis }\end{array}$ & $\mathbf{P} \mathbf{1}$ & $\mathbf{P} \mathbf{2}$ & $\mathbf{P 6}$ & $\mathbf{P 8}$ & $\mathbf{P 9}$ & $\mathbf{P 1 0}$ \\
\hline Dv4 & 5 & 0 & 10 & 0 & 5 & 5 \\
O v5 & 10 & 5 & 10 & 0 & 5 & 5 \\
Dv5 & 10 & 5 & 10 & 0 & 5 & 5 \\
O v6 & 15 & 10 & 15 & 0 & 10 & 5 \\
Dv6 & 15 & 15 & 15 & 0 & 10 & 5 \\
O v7 & 10 & 5 & 10 & 0 & 10 & 5 \\
Dv7 & 10 & 5 & 10 & 0 & 10 & 5 \\
O v8 & 15 & 10 & 15 & 0 & 10 & 10 \\
Dv8 & 15 & 10 & 15 & 0 & 10 & 10 \\
O v9 & 5 & 0 & 10 & 0 & 5 & 0 \\
Dv9 & 5 & 5 & 10 & 0 & 5 & 0 \\
O v10 & 0 & 0 & 5 & 0 & 0 & 0 \\
D v10 & 5 & 0 & 5 & 0 & 0 & 0 \\
O v11 & 75 & 40 & 100 & 5 & 60 & 45 \\
D v11 & 85 & 55 & 100 & 5 & 60 & 45 \\
\hline
\end{tabular}

Pelo teste de W ilcoxon, nos pacientes que não realizaram hidroterapia, as 5 primeiras variáveis, nos meses de outubro e dezembro foram de:

Tabela III - Teste deW ilcoxon para as 5 primeiras variáveis.

\begin{tabular}{cccccc}
\hline & $\begin{array}{c}\text { Dez V1 } \\
\text { O ut V1 }\end{array}$ & $\begin{array}{c}\text { Dez V2 } \\
\text { O ut V2 }\end{array}$ & $\begin{array}{c}\text { Dez V3 } \\
\text { O ut V3 }\end{array}$ & $\begin{array}{c}\text { Dez V4 } \\
\text { O ut V4 }\end{array}$ & $\begin{array}{c}\text { Dez V5 } \\
\text { O ut V5 }\end{array}$ \\
\hline $\begin{array}{l}\text { Z calculado } \\
\text { p } 000\end{array}$ &, 000 & $-1,000$ & $-1,000$ &, 000 \\
\hline & 1,000 & 1,000 & 0,317 & 0,317 & 1,000 \\
\hline
\end{tabular}

Pelo teste estatístico, tas médias das 5 primeiras variáveis dos pacientes que não realizaram hidroterapia são apresentadas na Tabela IV.

Tabela IV - M édias das variáveis 1 a 5, dos pacientes que não realizaram hidroterapia.

\begin{tabular}{llc}
\hline & N & Médias \\
\hline O ut V1 & 6 & 5,8 \\
O ut V2 & 6 & 2,5 \\
O ut V3 & 6 & 6,7 \\
O ut V4 & 6 & 3,3 \\
O ut V5 & 6 & 5,8 \\
Dez V1 & 6 & 5,8 \\
Dez V2 & 6 & 2,5 \\
Dez V3 & 6 & 7,5 \\
Dez V4 & 6 & 4,2 \\
Dez V5 & 6 & 5,8
\end{tabular}

Pelo teste de W ilcoxon, nos pacientes que não realizaram hidroterapia, as 6 últimas variáveis, nos meses de outubro e dezembro, são apresentadas na Tabela V.

Tabela $\mathbf{V}$ - TestedeW ilcoxon para as 6 últimas variáveis.

$\begin{array}{llllllll}\text { Dez V6 } & \text { Dez V7 } & \text { Dez V8 } & \text { Dez V9 } & \text { Dez V10 }\end{array}$

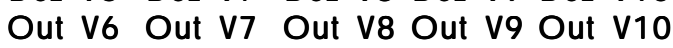

\begin{tabular}{lcllcc}
\hline$Z$ calculado & -1 & 0 & 0 & -1 & -1 \\
$\mathrm{p}$ & 0,317 & 1 & 1 & 0,317 & 0,317 \\
\hline
\end{tabular}

Pelo teste estatístico, as médias das 6 últimas variáveis dos pacientes que não realizaram hidroterapia são apresentadas na Tabela VI.

Tabela VI - M édias das variáveis 6 a 11, dos pacientes que não realizaram hidroterapia.

\begin{tabular}{llc}
\hline & N & Médias \\
\hline O ut V6 & 6 & 9,167 \\
O ut V7 & 6 & 6,667 \\
O ut V8 & 6 & 10,000 \\
O ut V9 & 6 & 3,333 \\
O ut V10 & 6 &, 833 \\
O ut V11 & 6 & 54,167 \\
Dez V6 & 6 & 10,000 \\
Dez V7 & 6 & 6,667 \\
Dez V8 & 6 & 10,000 \\
Dez V9 & 6 & 4,167 \\
Dez V10 & 6 & 1,667 \\
Dez V11 & 6 & 58,333 \\
\hline
\end{tabular}

A partir dos escores obtidos com o Índice de Barthel, no mês de outubro e dezembro, dos pacientes que realizaram terapia no solo mais a hidroterapia $(S=1)$, temos os seguintes valores das variáveis (Tabela VII).

T abela VII - R esultados do I ndice de Barthel para cada variável em outubro e dezembro dos pacientes que fizeram hidroterapia.

\begin{tabular}{lccccc}
\hline $\begin{array}{l}\text { Pacientes } \\
\text { Variáveis }\end{array}$ & P3 & P4 & P5 & P 7 & P1 1 \\
\hline O v1 & 5 & 10 & 10 & 5 & 5 \\
D v1 & 5 & 10 & 10 & 5 & 5 \\
O v2 & 5 & 0 & 5 & 5 & 0 \\
D v2 & 5 & 5 & 5 & 5 & 0 \\
O v3 & 10 & 10 & 10 & 10 & 10 \\
D v3 & 10 & 10 & 10 & 10 & 5 \\
O u4 & 10 & 10 & 10 & 10 & 5 \\
Dv4 & 10 & 10 & 10 & 10 & 10 \\
O v5 & 5 & 10 & 10 & 10 & 5 \\
Dv5 & 10 & 10 & 10 & 10 & 5 \\
O v6 & 5 & 15 & 15 & 15 & 10 \\
Dv6 & 5 & 15 & 15 & 15 & 10 \\
O v7 & 10 & 10 & 10 & 10 & 10 \\
Dv7 & 10 & 10 & 10 & 10 & 10 \\
O v8 & 10 & 10 & 10 & 15 & 0 \\
D v8 & 10 & 15 & 10 & 15 & 10 \\
O v9 & 0 & 5 & 5 & 5 & 0 \\
D v9 & 0 & 10 & 5 & 5 & 10 \\
O v10 & 5 & 5 & 5 & 5 & 0 \\
D v10 & 5 & 5 & 5 & 5 & 0 \\
O v11 & 65 & 85 & 90 & 90 & 45 \\
\hline
\end{tabular}

Pelo teste de W ilcoxon, nos pacientes que realizaram terapia no solo mais hidroterapia, as 5 primeiras variáveis, nos meses de outubro e dezembro, são apresentadas $n$ Tabela VIII. 
Tabela VIII - Teste de W ilcoxon nos pacientes que realizaram hidroterapia com relação às 5 primeiras variáveis.

\begin{tabular}{lcccccc}
\hline \multicolumn{8}{r}{ DEZ V1 } & DEZ V2 & DEZ V3 & DEZ V4 & DEZ V5 \\
O UT V1 & OUT V2 & OUT V3 & O UT V4 & O UT V5 \\
\hline Z calculado & 0 & -1 & -1 & -1 & -1 \\
$\mathrm{p}$ & 1 & 0,317 & 0,317 & 0,317 & 0,317 \\
\hline
\end{tabular}

Pelo teste estatístico, as médias das 5 primeiras variáveis dos pacientes que realizaram terapia no solo mais a hidroterapia são apresentadas na Tabela IX.

T abela IX - M édias das variáveis 1 a 5 dos pacientes querealizaram hidroterapia.

\begin{tabular}{lcc}
\hline & N & Médias \\
\hline O ut V1 & 5 & 7,0 \\
O ut V2 & 5 & 3,0 \\
O ut V3 & 5 & 10,0 \\
O ut V4 & 5 & 9,0 \\
O ut V5 & 5 & 8,0 \\
Dez V1 & 5 & 7,0 \\
Dez V2 & 5 & 4,0 \\
Dez V3 & 5 & 9,0 \\
Dez V4 & 5 & 10,0 \\
Dez V5 & 5 & 9,0 \\
\hline
\end{tabular}

Pelo teste de Wilcoxon, nos pacientes que realizaram terapia no solo mais hidroterapia, as 6 últimas variáveis, nos meses de outubro e dezembro são apresentadas na Tabela $X$.

Tabela $\mathbf{X}$ - Teste de W ilcoxon nos pacientes que realizaram hidroterapia com relação às 6 últimas variáveis.

\begin{tabular}{lcccccc}
\hline \multicolumn{3}{r}{ DEZ V6 } & DEZ V7 & DEZ V8 & DEZ V9 & DEZ V10 \\
O UT V6 & O UT V7 & O UT V8 & O UT V9 & O UT V10 \\
\hline Z calculado & 0 & 0 & $-1,342$ & $-1,342$ & 0 \\
$\mathrm{p}$ & 1 & 1 & 0,18 & 0,18 & 1 \\
\hline
\end{tabular}

Pelo teste estatístico, as médias das 6 últimas variáveis dos pacientes que realizaram terapia no solo mais a hidroterapia são apresentadas na Tabela XI.

T abela XI - M édias das variáveis 6 a 11, dos pacientes querealizaram hidroterapia.

\begin{tabular}{lcc}
\hline & N & Médias \\
\hline O UT V6 & 5 & 12,0 \\
O UT V7 & 5 & 10,0 \\
O UT V8 & 5 & 9,0 \\
O UT V9 & 5 & 3,0 \\
O UT V10 & 5 & 4,0 \\
O UT V11 & 5 & 75,0 \\
DEZ V6 & 5 & 12,0 \\
DEZ V7 & 5 & 10,0 \\
DEZ V8 & 5 & 12,0 \\
DEZ V9 & 5 & 6,0 \\
DEZ V10 & 5 & 4,0 \\
DEZ V11 & 5 & 83,0 \\
\hline
\end{tabular}

Para compararmos possíveis diferenças no resultado final entre os grupos sem hidroterapia e com hidroterapia para todas as variáveis, usando-se a variável previamente definida (delta \%), os resultados são apresentaos na Tabela XII.

\section{Discussão}

Com relação ao grupo de pacientes sem hidroterapia, as variáveis V 1, V2 e V 5 (alimentação, asseio, vestir), no mês de outubro, apresentaram médias, respectivamente, de 5,8; 2,5 e 5,8. Em dezembro, estas médias se mantiveram com o mesmo valor. Observa-se que não houve uma diferença estatisticamente significativa, sendo o Z calculado $=0,00(p=1,00)$, o que mostra evidentemente que não houve uma diferença estatisticamente significativa (Tabelas III e IV).

Tabela XII - Testede M ann-W hitney (delta \%) com as variáveis V 1 a V 5.

\begin{tabular}{lccccc}
\hline Hidro & DELTA V1 & DELTAV2 & DELTAV3 & DELTAV4 & DELTAV5 \\
\hline Sem N Mean & 60,0 & 30,0 & 520,0 & 30,0 & 50,0 \\
Com N Mean & 50,0 & 30,0 & $5-10,0$ & 520,0 & 520,0 \\
Teste de Mann-Whitney & & & & & $-1,000$ \\
Z calculado & 0,000 & 0,000 & $-1,342$ & $-0,775$ & 0,690 \\
P & 1,000 & 1,000 & 0,421 & 0,786 & \\
\hline
\end{tabular}

Tabela XIII - Teste de M ann-W hitney (delta \%) com as variáveis V 6 a V 11.

\begin{tabular}{lcccccc}
\hline Hidro & DELTA V6 & DELTA V7 & DELTA V8 & DELTAV9 & DELTAV10 & DELTA V11 \\
\hline Sem N Média & 510,0 & 50,0 & 50,0 & 30,0 & 10,0 & 68,5 \\
Com N Média & 50,0 & 50,0 & 412,5 & 333,3 & 40,0 & 513,9 \\
Teste de Mann-Whitney & & & & & & $-1,000$ \\
Z calculado & $-1,000$ & 0,000 & $-1,118$ & 0,000 &,- 796 \\
p & 0,690 & 1,000 & 0,556 & 0,700 & 1,000 & 0,537 \\
\hline
\end{tabular}


A variável V3 (evacuação), no mês de outubro, apresentou média de 6,7. Em dezembro, esta média foi de 7,5. Observa-se que não houve uma diferença estatisticamente significativa, $Z$ calculado $=-1,000(p=0,317)$, embora tenha havido uma melhora nesta variável nos pacientes que só realizaram terapia no solo (Tabelas III e IV).

A variável V4 (micção), no mês de outubro, apresentou média de 3,3. Em dezembro, está média foi de 4,2. O bservase que não houve uma diferença estatisticamente significativa, $Z$ calculado $=-1,000(p=0,317)$, mas nota-se que ocorreu um aumento na média desta variável nos pacientes que só realizaram terapia no solo (Tabelas III e IV).

As variáveis $\mathrm{V} 7$ e V8 (toalete e mobilidade), no mês de outubro, apresentaram médias, respectivamente, de 6,7 e 10,0. E m dezembro, estas médias se mantiveram com mesmo valor. Observa-se que não houve uma diferença estatisticamente significativa, Z calculado $=0,00(p=1,00)$ (Tabelas V e VI).

A varíável V6 (cadeira/cama), no mês de outubro, apresentou média de 9,2, e em dezembro esta média foi igual a 10,0 . Nota-se que não houve uma diferença estatisticamente significativa, $Z$ calculado $=-1,00(p=$ $0,317)$, mas ocorreu aumento na média desta variável nos pacientes que só realizaram terapia no solo (Tabelas V e VI).

A variável $V 9$ (escadas), no mês de outubro, apresentou média de 3,3, e em dezembro esta média foi igual a 4,2. N ão houve uma diferença estatisticamente significativa, $Z$ calculado $=-1,00(p=0,317)$, embora tenha havido uma melhora nesta variável nos pacientes que só realizaram terapia no solo (Tabelas V e VI).

A variável V10 (banho), no mês de outubro, mostrou média de 0,8 , e em dezembro esta média foi igual a 1,7 . N ão houve uma diferença estatisticamente significativa, $Z$ calculado $=-1,00(p=0,317)$, mas nota-se que ocorreu um aumento na média desta variável (Tabelas $\mathrm{V}$ e VI).

A variável V11 (total), no mês de outubro, apresentou média de 54,2, e em dezembro esta média foi igual a 58,3. $N$ ão houve uma diferença estatisticamente significativa, $Z$ calculado $=-1,342(p=0,180)$, mas nota-se que ocorreu uma melhora desta variável nos pacientes que somente realizaram terapia no solo (Tabelas V e VI).

Quanto ao grupo tratado com a terapia no solo e com a hidroterapia, nota-se que houve melhora nas seguintes variáveis; V2, V4, V5, V8, V9 e V11.

A variável V2 (asseio), no mês de outubro, apresentou média de 3,0. Em dezembro, esta média foi de 4,0. O bservase que não houve diferença estatisticamente significativa, $Z$ calculado $=-1,00(p=0,317)$, mas ocorreu uma melhora na média dessa variável após o paciente ter realizado terapia no solo mais hidroterapia (Tabelas VIII e IX).

A variável V4 (micção), no mês de outubro, apresentou média de 9,0. Em dezembro, esta média foi de 10,0. O bservase que não houve diferença estatisticamente significativa, $Z$ calculado $=-1,00(p=0,317)$, mas ocorreu um aumento na média dessa variável (Tabelas VIII e IX).

A variável V5 (vestir), no mês de outubro apresentou média de 8,0. Em dezembro, esta média passou para 9,0. $N$ ão houve diferença estatisticamente significativa, $Z$ calculado $=-1,00(p=0,317)$, embora tenha havido melhora nesta média após as terapias no solo mais hidroterapia (Tabelas VIII e IX).

A variável $\mathrm{V} 8$ (mobilidade), no mês de outubro, mostrou média de 9,0. Em dezembro, esta média subiu para 12,0. $\mathrm{N}$ ão houve diferença estatisticamente significativa, $\mathrm{Z}$ calculado $=-1,342(p=0,180)$, mas ocorreu aumento na média dessa variável (Tabelas $\mathrm{X}$ e XI).

$\mathrm{N}$ a variável $\mathrm{V} 9$ (escadas), no mês de outubro, a média foi de 3,0 e em dezembro esta média foi igual a 6,0. Não houve diferença estatisticamente significativa, $Z$ calculado igual a $-1,342(p=0,180)$, embora tenha havido um aumento na média dessa variável (Tabelas $X$ e XI).

$\mathrm{N}$ a variável V11 (total), no mês de outubro, a média foi de 75,0 e em dezembro, esta média foi igual a 83,0. N ão houve diferença estatisticamente significativa, $Z$ calculado igual a - 1,604 ( $p=0,109)$, mas nota-se um aumento nesta média (Tabelas $X$ e XI).

$\mathrm{N}$ a tabela XII, observa-se que em relação a variável $\mathrm{V} 3$, a média no grupo sem hidroterapia foi de $20,0 \%$, enquanto que no grupo com hidroterapia a média foi de $-10,0 \%$. Isso significa que, para esta variável, o percentual de melhora no grupo sem hidroterapia foi $20 \%$ em média, enquanto que no grupo com hidroterapia esta variável foi de $-10 \%$, ou seja, houve uma pequena piora. Entretanto, não houve uma diferença estatisticamente significativa pois a estatística calculada foi igual a - 1,342 ( $p=0,421)$.

Q uanto à variável $V 4$, nota-se que a média no grupo sem hidroterapia foi de $0,0 \%$, enquanto que no grupo com hidroterapia a média foi de $20,0 \%$. Isso significa que para esta variável o percentual de melhora no grupo com hidroterapia foi de $20 \%$ em média, mostrando, entretanto, não haver uma diferença estatisticamente significativa, pois a estatística calculada foi igual a $-0,775$; com $p=0,786$ (Tabela XII).

Quanto à variável $\mathrm{V} 5$, também se nota que a média no grupo sem hidroterapia foi de $0,0 \%$, enquanto que no grupo com hidroterapia a média foi de $20,0 \%$. Isso mostra que para esta variável o percentual de melhora no grupo com hidroterapia foi de $20 \%$ em média, observando-se, entretanto, não haver uma diferença estatisticamente significativa, pois a estatística calculada foi igual a $-1,00$, sendo o $p=0,690$ (Tabela XII).

$\mathrm{N}$ a variável $\mathrm{V} 6$, no grupo sem hidroterapia, houve uma melhora de $10 \%$ enquanto que no grupo com hidroterapia não se observou nenhuma melhora (Tabela XIII).

Quanto à variável $V 8$, verifica-se que a média no grupo sem hidroterapia foi de $0,0 \%$, enquanto que no grupo com hidroterapia, foi de $12,5 \%$. Ou seja, para esta variável o 
percentual de melhora no grupo com hidroterapia foi de $12,5 \%$ em média, embora não tenha havido uma diferença estatisticamente significativa, pois a estatística calculada foi igual a $-1,118$, sendo o $p=0,556$ (Tabela XIII).

Quanto à variável $V 9$, nota-se que a média no grupo sem hidroterapia foi de $0,0 \%$, enquanto que no grupo com hidroterapia, a média foi de $33,3 \%$. Isso significa que para esta variável o percentual alto de melhora no grupo com hidroterapia foi de 33,3\% em média, mostrando, entretanto, não haver uma diferença estatisticamente significativa, pois a estatística calculada foi igual a $-1,00$ e o $p=0,70$ (Tabela XIII).

Quanto à variável V11, nota-se que a média no grupo sem hidroterapia foi de 8,5\%. Já no grupo com hidroterapia, a média foi de $13,9 \%$. N ão houve diferença estatisticamente significativa, pois a estatística calculada foi igual a -0,796 e o $p=0,537$, mas ocorreu um aumento na média na variável total (Tabela XIII).

\section{Conclusão}

A pesar do trabalho não apresentar, dentre as variáveis, nenhuma diferença estatisticamente significativa, verificouse que houve melhora em algumas variáveis após 2 meses de fisioterapia, tanto no grupo onde os pacientes só realizaram terapia no solo, quanto no grupo em que os pacientes foram submetidos à terapia no solo mais hidroterapia.

Os pacientes que não realizaram hidroterapia apresentaram aumento nas médias das variáveis: evacuação, micção, cadeira/ cama, escadas, banho e na variável total.

Os pacientes que realizaram hidroterapia, associada à terapia no solo, apresentaram melhora nas médias das variáveis: asseio, micção, vestir, mobilidade, escadas e na variável total.

Se compararmos as diferenças das médias da variável total de outubro e dezembro entre os pacientes que não fizeram hidroterapia e os que fizeram hidroterapia, perceberemos que houve uma variação de 4,1 nos pacientes que não fizeram hidro, enquanto que, nos pacientes que fizeram hidroterapia, essa variação foi maior: 8,0. Isso sugere que os pacientes que associam a terapia no solo à hidroterapia têm maior probabilidade de melhorar as AVDs do que aqueles que só realizam a terapia no solo.

Esse trabalho demonstra que, mesmo em um curto período de tempo de 2 meses, os pacientes que sofreram um acidente vascular encefálico podem apresentar uma maior facilidade em realizar suas atividades de vida diária devido à prática da fisioterapia, mesmo que sua reabilitação completa só seja conquistada após anos de tratamento fisioterapêutico.

\section{Referências}

1. Sacco RL. Patogênese, classificação e epi demiologia das doenças vasculares cerebrais.

2. In: Rowland LP, M errett. Tratado de neurologia. 9a ed. Rio de J aneiro: G uanabara K oogan; 1989. p.177-90.

3. Pereira CF, L emos M M , Benvenuto M C, Fonseca G A. Enfoque sobre a pesquisa prospectiva no AVC. M ed Reabil 1993;34:9-13.

4. Chagas EF, Tavares M CG CF. Simetria e transferência de peso do hemiplégico: relação dessa condição com o desempenho de suas atividades funcionais. R ev F isioter U niv São Paulo 2001;8(1):4050 .

5. Christiansen $\mathrm{CH}$, Schwartz K, Barnes KJ. Self-care: evaluation and management. In: D eL isaJ A (ed). Rehabilitation medicine: principles and practice. Philadelphia: J B L ippincott; 1988.

6. Aitken MJ. Self-concept and functional independence in the hospitalized elderly. A m J O ccup Ther 1982;36:243-50.

7. M alick M H, A Imasy B. A ssessment and evaluation-lifeworks tasks. In: Hopkins HL, Smith HD (eds). W illard and Spackman's occupational therapy. 6a ed. Philadel phia: J B L ippincott; 1983.

8. Slatter SB, Sussman M B, Stroud M W. Participation in household activities as prognostic factor for rehabilitation. A rch Phys $M$ ed Rehabil 1970;51(10):605-10.

9. Bartels EC. A contemporary framework for independent living rehabilitation. Rehabil L it 1985;46:325-7

10. Jacelon CS. The Barthel Index and other indices of functional ability. Rehabil N urs 1986;11(4):9-11.

11. Van der Putten JJ M F, H obart J C, Freeman JA, Thompson AJ. $M$ easuring change in disability after inpatient rehabilitation: comparison of the responsiveness of the Barthel Index and the functional independence measure. J N eurol N eurosurg P sychiatry 1999;66:480-4.

12. Roy CW, Togneri J, H ay E. Pentland B. An inter-rater reliability study of the Barthel Index. Int J Rehabil Res 1988;11:67-70.

13. M CG innis GE, Seward ML, Dejong G, O sberg JS. Program evaluation of physical medicineand rehabilitation departmentsusing self-report Barthel. A rch Phys M ed Rehabil 1986;67:123-5.

14. Roden-J ulling A, Britton M, G ustafsson C, F ugl- M eyer A. Validation of four scales for theacutestage of stroke. J Intern $M$ ed 1994;236:125-36.

15. Siegel S. Estatística não-paramétrica (para as ciências do comportamento). São Paulo: M anole; 1999. 